\title{
Single-Molecule Approach to DNA Minor-Groove
} Association Dynamics

Jorge Bordello, Mateo I. Sánchez, M. Eugenio Vázquez, José L. Mascareñas, Wajih Al-Soufi, Mercedes Novo

\section{Peer reviewed version}

This is the peer reviewed version of the following article: Bordello, J. , Sánchez, M. I., Vázquez, M. E., Mascareñas, J. L., Al-Soufi, W. and Novo, M. (2012), Single-Molecule Approach to DNA MinorGroove Association Dynamics. Angew. Chem. Int. Ed., 51: 7541-7544, which has been published in final form at https://doi.org/10.1002/anie.201201099. This article may be used for non-commercial purposes in accordance with Wiley Terms and Conditions for Use of Self-Archived Versions

\section{How to cite:}

Bordello, J., Sánchez, M. I., Vázquez, M. E., Mascareñas, J. L., Al-Soufi, W. and Novo, M. (2012), Single-Molecule Approach to DNA Minor-Groove Association Dynamics. Angew. Chem. Int. Ed., 51: 7541-7544. doi:10.1002/anie.201201099

\section{Copyright information:}

(C) 2012 WILEY-VCH Verlag GmbH \& Co. KGaA, Weinheim. This article may be used for noncommercial purposes in accordance with Wiley Terms and Conditions for Use of Self-Archived Versions 


\section{Single-Molecule Approach to DNA Minor-Groove Association Dynamics}

Jorge Bordello ${ }^{l}$,Mateo I. Sánchez ${ }^{2}$, M. Eugenio Vázquez ${ }^{2}$, José L. Mascareñas ${ }^{2}$, Wajih Al-Soufi ${ }^{l}$ and Mercedes Novo ${ }^{*}$

Chemists have long pursued the design and preparation of molecules that can recognize specific DNA sequences. The deciphering of the human genome and on-going efforts to sequence the genome of many other organisms have provided a wealth of information on DNA targets of both therapeutic and diagnostic interest, and therefore there is a renewed interest in the development of smart DNA minor-groove binders. ${ }^{[1,2]}$ Despite the detailed structural and thermodynamic information available regarding the interaction of a number of minor-groove binders with the DNA, kinetic data are much more scarce. In addition, the very low dissociation rates typical of these agents result in very slow binding dynamics, which are experimentally not easily accessible. ${ }^{[3]}$ Typically used stoppedflow methods can yield complex kinetics and artifacts arising from the relatively high binder concentrations often required in this technique. Moreover, the dissociation rates are usually obtained with indirect SDS sequestering techniques that only give apparent rates. Dynamic data of archetypical minor-groove binders such as Distamycin or Hoechst 33258 indicate that the association process is very fast and nearly diffusion limited, which is surprising given the severe geometric constraints imposed on the inclusion of a binder into the narrow minor groove. ${ }^{[4-7]}$

Cationic bis-benzamidines represent interesting platforms to target A/T-rich sequences preferentially over those containing $\mathrm{G} / \mathrm{C}$ pairs. Prominent examples of this family, such as pentamidine or furamidine, have found clinical applications and are successfully used for the treatment of several major tropical diseases. ${ }^{[8]}$ Nevertheless, the toxic side effects of these bis-benzamidines ${ }^{[9]}$ have encouraged the search for new derivatives with improved efficacy, pharmacological properties and reduced adverse effects. ${ }^{[10]}$ Bis-

[*] J. Bordello, Prof. Dr. W. Al-Soufi and Prof. Dr. M. Novo* Departamento de Química Física Universidade de Santiago de Compostela Facultade de Ciencias, 27001 Lugo (Spain) Fax: $(+) 34982824001$

E-mail:m.novo@usc.es

\section{I. Sánchez, Prof. Dr. M. E. Vázquez, Prof. Dr. J. L.} Mascareñas

Departamento de Química Orgánica y Centro Singular de Investigación en Química Biológica y Materiales

Moleculares, Unidad Asociada al CSIC

Universidade de Santiago de Compostela

15782 Santiago de Compostela (Spain)

[**] The authors thank the Ministerio de Ciencia e Innovación (CTQ2010-21369, SAF2007-61015, SAF2010-20822-C02, CTQ2009-14431/BQU, Consolider Ingenio 2010 CSD200700006) and the Xunta de Galicia (INCITE09262304PR, INCITE09E2R209064ES, IN845B-2010/094, INCITE09 209 084PR, GRC2010/12, PGIDIT08CSA-047209PR) for financial support. J.B. and M.I.S thank the Ministerio de Ciencia e Innovación for their research scholarships.

Supporting information for this article is available on the WWW under http://www.angewandte.org or from the author. benzamidines bind short $\mathrm{A} / \mathrm{T}$-rich DNA sequences by insertion in the narrow minor groove. NMR and $\mathrm{X}$-ray studies agree in the general picture of the interaction, in which the positively charged amidinium groups are situated deep in the groove, making both direct and indirect hydrogen bonds with the DNA bases and electrostatic contacts with the bottom of the groove. ${ }^{[11]}$

Recently we reported several rapid and practical synthetic approaches to bis-benzamidine DNA binders, some of which can be easily modified by conjugation to designed functionalities or fluorophores. ${ }^{[12]}$ With this methodology at hand we decided to make the conjugate BBA-OG (Figure 1), featuring an Oregon green fluorophore attached to the DNA binder. We envisioned that the presence of a negative carboxylate charge in the fluorophore should increase the DNA dissociation rate, and thereby allow us to extract kinetic information using Fluorescence Correlation Spectroscopy (FCS). This technique provides a sensitive single-molecule approach to study fast kinetics of biomolecular interactions, ${ }^{[13]}$ yielding accurate values for the binding equilibrium constant and the association and dissociation rate constants, as well as for the diffusion coefficients, ${ }^{[14,15]}$ from which the diffusion-controlled rate constants can be estimated. ${ }^{[16]}$ To the best of our knowledge this technique has not been previously used to analyze the dynamics of minor groove binders. The wide dynamic range of FCS makes it possible to identify the rate-limiting step in the association event by comparison of the dynamics of the association to DNA with different sequences and chain length, and thus to evaluate the importance of processes such as sliding or two-dimensional diffusion, which have been proposed as likely mechanisms for the binding of proteins to DNA. ${ }^{[16,17]}$

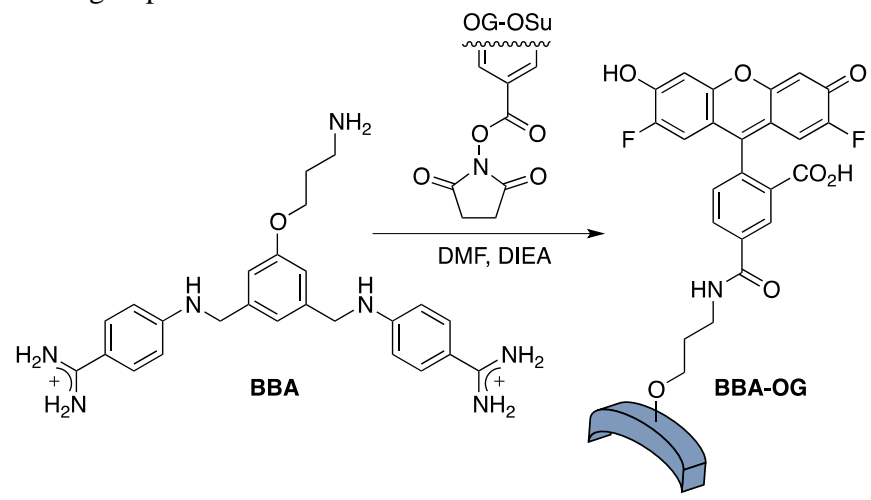

Figure 1. Synthesis of Oregon green-labeled bis-benzamidine BBAOG.

FCS titrations of BBA-OG with three short hairpin oligonucleotides (12 bp + loop) containing as key target sequences $A A A T T T, A A T T T$ and GGCCC and with a longer dsDNA (50 bp) containing one AAATTT site, were performed. For the AAATTT-hp an additional FCS titration at a higher salt concentration was measured. Figure 2 shows normalized fluorescence correlation curves of the binding of BBA-OG to the longer dsDNA. Without 
DNA only translational diffusion of the binder with correlation time $\tau_{D}$ is observed. Addition of DNA increases $\tau_{D}$ reflecting the slower diffusion of the dye bound to DNA. Additionally a new term appears with a shorter correlation time $\tau_{R}$, which decreases as the concentration of DNA is increased (see insets in Figure 1). $\tau_{R}$ is the relaxation time of the reversible binding process: $\tau_{R}=k_{+}[\mathrm{DNA}]+k_{-}^{-1}$ with $k_{+}$and $k_{-}$being the association and dissociation rate constants, respectively. The huge amplitude $A_{R}$ of this term is due to the high increase in the brightness of bound BBAOG. ${ }^{[18]}$ The diffusion, and relaxation terms show the expected concentration dependence and are independent of the irradiance. ${ }^{[18,19]}$ In order to reduce parameter correlation, the FCS titration series were analyzed by global target fits ${ }^{[14,15]}$ with a common dynamic-equilibrium model of the diffusion, triplet and relaxation terms (Figure 2 and SI.1 to SI.4). The parameters that best fit the whole data set are given in Table 1 and Figure 3.

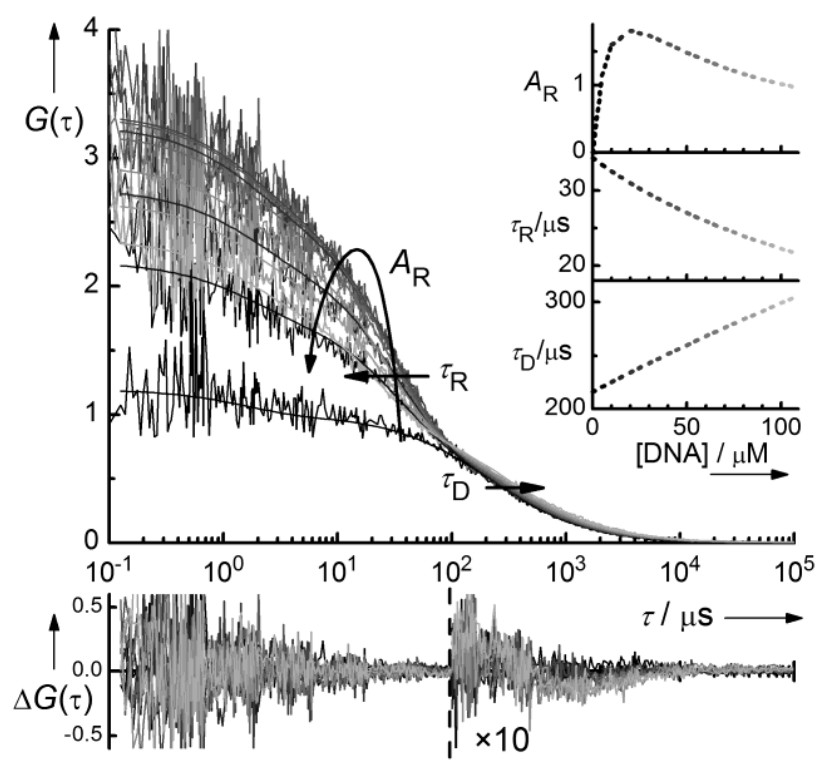

Figure 2.FCS curves of the titration of BBA-OG with AAATTT-ds and results of a global fit of the dynamic-equilibrium model, eq. SI.1-4. Insets: variation of the mean diffusion time $\bar{\tau}_{D}$, the relaxation time $\tau_{R}$ and the relaxation amplitude $A_{R}$ with [DNA] calculated from the fit parameters. Lower panel: Unweighted fit residuals. The small deviations at high DNA concentrations are due to minor fluorescent impurities in the DNA samples. Above $100 \mu$ s a factor of 10 was applied.

Table 1. Association $\left(k_{+}\right)$and dissociation $(k)$ rate constants and binding equilibrium constant $(K)$ of BBA-OG with dsDNA. The values of $k_{+}$are calculated from the fit parameters $k$. and $K\left(K=k_{+} / k_{-}\right)$.

\begin{tabular}{llll}
\hline DNA $^{[a]}$ & $k_{+} / 10^{8} \mathrm{M}^{-1} \mathrm{~s}^{-1}$ & $k_{-} / 10^{4} \mathrm{~s}^{-1}$ & $K / 10^{3} \mathrm{M}^{-1}$ \\
\hline AAATTT-hp $^{[a]}$ & $1.36 \pm 0.01$ & $3.19 \pm 0.02$ & $4.27 \pm 0.03$ \\
AATTTT-hp $^{[a]}$ & $0.95 \pm 0.01$ & $4.89 \pm 0.05$ & $1.95 \pm 0.02$ \\
GGCCC-hp $^{[a]}$ & $0.083 \pm 0.003$ & $14.0 \pm 0.4$ & $0.059 \pm 0.001$ \\
AAATTT-ds $^{[a]}$ & $1.56 \pm 0.02$ & $2.84 \pm 0.02$ & $5.47 \pm 0.05$ \\
AAATTT-hp $^{[b]}$ & $0.65 \pm 0.03$ & $4.63 \pm 0.01$ & $1.41 \pm 0.03$ \\
\hline
\end{tabular}

[a] $[\mathrm{NaCl}]=0.1 \mathrm{M},[\mathrm{b}][\mathrm{NaCl}]=1.0 \mathrm{M}$. See complete oligonucleotide sequences in the SI.

Figure 3 shows that the association rate constant decreases by more than one order of magnitude from the AT rich to GC containing sequences, whereas the dissociation rate constant increases 3-5 times. Both association and dissociation contribute to the specificity for the AT rich tracts, but the association has a higher effect. This is in agreement with reported results for the antibiotic Distamycin with different mismatched sites. ${ }^{[4]}$ Moreover, the absolute values of $k_{+}$for the AT sequences coincide well with those reported for other minor-groove binders ${ }^{[4-7]}$ and show the small decrease with $\mathrm{Na}^{+}$concentration expected for groove binders, ${ }^{[20]}$ suggesting analogous association mechanisms. Nevertheless, the dissociation rate constants are about 4 orders of magnitude higher than those of typical binders, resulting in much lower binding constants.

The above results indicate much weaker specific interactions of BBA-OG with the DNA minor groove, which is probably due to the electrostatic repulsion between the negatively charged OG moiety and DNA. The high binding constants of unlabeled bisbenzamidines supports this explanation. ${ }^{[12]}$ On this basis we can consider BBAOG as a valid model for the study of the association process to the minor groove of the DNA.

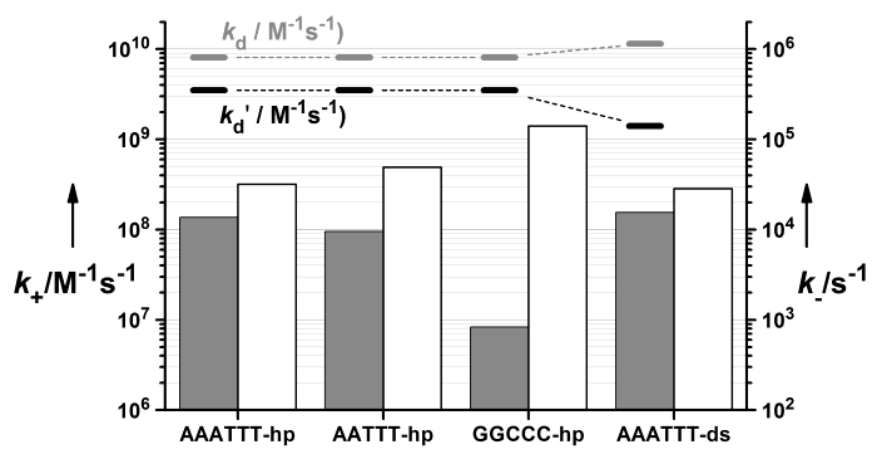

Figure 3.Association (grey, left scale) and dissociation (white, right scale) rate constants of BBA-OG with DNA obtained from FCS.

Estimates of the diffusion-controlled association rate contants $k_{d}$ and $k_{d}^{\prime}$ defined in Scheme 1 (see SI).

In order to determine the rate-limiting step for the association process we compare the dynamics of binding to the short DNA AAATTT-hp with that to the much longer AAATTT-ds, which has been designed avoiding other AT rich sites. Curiously, we don't find significant differences in the binding dynamics (Table 1, Figure 3). The similar dissociation $\left(k_{-}\right)$is readily explained in terms of similar specific interactions with the AAATTT sequence. However, if diffusion plays an important role, the association dynamics should be affected by the increase of the DNA length.

For a more detailed analysis we propose a two-step mechanism like that previously used to describe other supramolecular binding processes (Scheme 1). ${ }^{[14,15]}$ The first step is the formation of an encounter complex between DNA and binder, with a diffusioncontrolled association rate which can be estimated based on the geometry of DNA and binder. The second step is the insertion of the binder into the minor-groove with a rate constant $k_{\mathrm{r}}$, a process which conveys structural rearrangements or the breakdown and reconstitution of the water structure around the interacting species. The experimentally observed $k_{+}$corresponds to the overall reaction described by a single relaxation time and is determined by the ratelimiting step. This mechanism is therefore different to the sequential model proposed for the binding of Hoechst 33258 to $\mathrm{DNA}^{[7]}$ which involves two reactions with their corresponding two observed reaction times. 
Assuming a rod-like structure of the DNA with a localized reactive site, we estimate lower bounds (see SI) of the diffusioncontrolled association rate constants $k_{\mathrm{d}}$ and $k_{\mathrm{d}}$ ' for the formation of unlocalized (U) and localized (L) encounter complexes, respectively, where the binder is either located arbitrarily at any position of the DNA (U) or already localized in the neighborhood of the reactive site (L) (Scheme 1). ${ }^{[16]}$

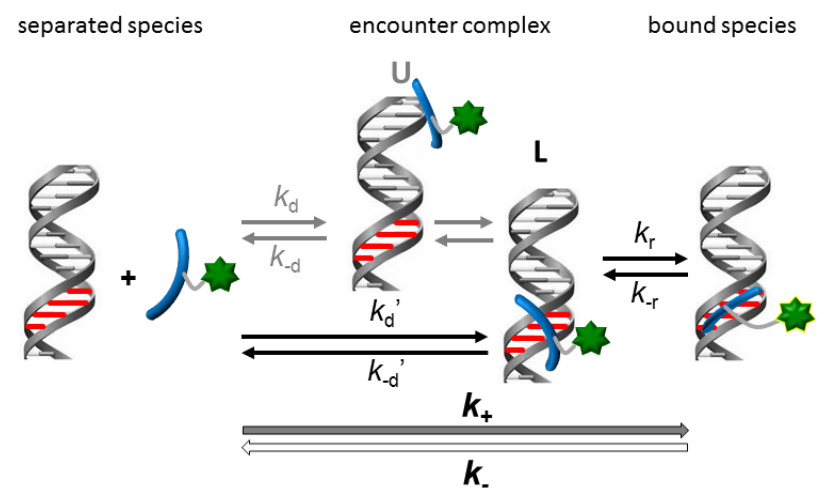

Scheme 1. Two-step mechanism proposed for the binding of BBAOG with DNA involving the formation of an encounter complex, with the binder localized at the DNA reactive site $(L)$ or unlocalized $(U)$.

The estimated values of $k_{\mathrm{d}}$ ' for the formation of localized complexes L are of course significantly lower than those of $k_{\mathrm{d}}$ corresponding to the formation of $\mathrm{U}$, especially in the case of the long dsDNA (Figure 3, SI). Nevertheless, even for the most specific sequences, both $k_{\mathrm{d}}$ and $k_{\mathrm{d}}$ ' are at least one order of magnitude higher than the observed overall rate constant $k_{+}$. This shows that the diffusion-controlled formation of the encounter complex is not the rate-limiting step, even if the binder is requested to be located near the reactive sequence. The encounter complexes $\mathrm{U}$ and $\mathrm{L}$ are in rapid pre-equilibrium with the separated species so that the overall rate of association between BBA-OG and DNA is not diffusion limited but determined by the next step of the process, namely the unimolecular inclusion into the minor groove. This step is probably essentially controlled by geometrical and orientational requirements, which depend on critical dimensions of both the binder and the minor groove. The presence of the exocyclic 2-amino group in GC tracts $^{[21]}$ may explain the much lower value of $k_{+}$obtained for this sequence.

We can also conclude that processes that increase the rate of formation of localized encounter complexes, such as sliding or bidimensional diffusion along the DNA strand, ${ }^{[16,17]}$ do not play a key role in the association rate of BBA-OG, and probably also not for other minor groove binders.

In summary, our results indicate that the association process of BBA-OG to dsDNA is not controlled by diffusion, but by the ratelimiting insertion of the binder into the minor-groove. Moreover, we find that the association process has an important effect on the specificity to AT sites, whereas the differences in the binding affinity are mainly determined by the dissociation rate. This mechanism might constitute a general mechanism for small minor groove binders, but this should be confirmed with further studies on more typical minor groove agents. This information should be useful for the design of new DNA binders with optimized properties and for the future understanding of the behavior of these molecules in more complex cellular environments. This work has also shown the potential of FCS for the study DNA binding dynamics of minorgroove binders using labeled derivatives.

\section{Experimental Section}

Oligonucleotides were purchased from Thermo Fisher Scientific Inc. Nucleotide sequences and the procedure for reconstitution and annealing are provided in the SI. Details of the synthesis of the BBA-OG can be found in the SI. Experimental setup and conditions, as well as the details of the data analysis for the FCS measurements have been published elsewhere ${ }^{[18,22]}$ and are specified for these experiments in the SI. The uncertainties given in Table 1 represent one standard deviation as obtained in the fits.

Received: ((will be filled in by the editorial staff))

Published online on ((will be filled in by the editorial staff))

Keywords: DNA minor-groove binder - binding dynamics .

Fluorescence Correlation Spectroscopy · DNA · Molecular recognition

[1] a) D. R. Boer, A. Canals, M. Coll, J. Chem. Soc., Dalton Trans. 2009, 3, 399-414; b) W. C. Tsee, D. L. Boger, Chem. Biol. 2004, 11, 16071617; c) E. Pazos, J. Mosquera, M. E. Vázquez, J. L. Mascareñas, ChemBioChem 2011, 12, 1958-1973. d) M. E. Vázquez, A. M. Caamaño and J. L. Mascareñas, Chem. Soc. Rev. 2003, 32, 338-349

[2] a) M. E. Vázquez, A. M. Caamaño, J. Martínez-Costas,L. Castedo, J. L. Mascareñas, Angew. Chem. Int. Ed. 2001, 40, 4723-4725; b) O. Vázquez, M. E. Vázquez, J. B. Blanco, L. Castedo, J. L. Mascareñas, Angew. Chem. Int. Ed. 2007, 46, 6886-6890.

[3] a) L. M. Wilhelmsson, P. Lincoln, B. Nordén in Sequence-Specific DNA Binding Agents (Ed:. M. Waring), The Royal Society of Chemistry 2006, pp. 69-95; b) T. C. S. Pace, C. Bohne, Adv. Phys. Org. Chem. 2007, 42, 167-223.

[4] R. Baliga, D. M. Crothers, J. Am. Chem. Soc. 2000, 122, 11751-11752.

[5] R. Baliga, D. M. Crothers, PNAS 2000, 97, 7814-7818.

[6] S. Y. Breusegem, S. Sadat-Ebrahimi, K. T. Douglas, R. M. Clegg, F. G. Loontiens, J. Mol. Biol. 2001, 308, 649-663.

[7] S. Y. Breusegem, R. M. Clegg, F. G. Loontiens, J. Mol. Biol. 2002, 315 , 1049-1061

[8] a) R. Balaña-Fouce, M. C. Redondo, Y. Pérez-Pertejo, R. Díaz-González, M. R. Reguera, Drug Discov. Today 2006, 11, 733-740; b) I. Midgley, K. Fitzpatrick, L. M. Taylor, T. L. Houchen, S. J. Henderson, S. J. Wright, Z. R. Cybulski, B. A. John, A. McBurney, D. W. Boykin, K. L. Trendler, Drug Metab. Dispos. 2007, 35, 955-967.

[9] a) W. D. Wilson, B. Nguyen, F. A. Tanious, A. Mathis, J. E. Hall, C. E. Stephens, D. W. Boykin, Curr. Med. Chem. Anticancer Agents 2005, 5, 389-408; b) A. H. Fairlamb, Trends Parasitol. 2003, 19, 488-494.

[10] R. R. Tidwell, D. W. Boykin in DNA and RNA Binders: From Small Molecules to Drugs (Eds.: M. Demeunynck, C. Bailly, W. D. Wilson), Wiley-VCH, Weinheim, Germany, 2003, pp. 414-460.

[11] C. M. Nunn, S. Neidle, J. Med. Chem. 1995, 38, 2317-2325.

[12] a) O. Vázquez, M. I. Sánchez, J. Martínez-Costas, M. E. Vázquez, J. L. Mascareñas, Org. Lett. 2010, 12, 216-219; b) O. Vázquez, M. I. Sánchez, J. L. Mascareñas, M. E. Vázquez, Chem. Commun. 2010, 46, 5518-5520; c) M. I. Sánchez, O. Vázquez, J. Martínez-Costas, M. E. Vázquez, J. L. Mascareñas, submitted.

[13] a) A. van Oijen, Curr. Opin. Biotechnol. 2011, 22, 75-80; b) D. Magde, E. L. Elson, W. W. Webb, Biopolymers 1974, 13, $29-61$.

[14] W. Al-Soufi, B. Reija, M. Novo, S. Felekyan, R. Kühnemuth, C. A. M. Seidel, J. Am. Chem. Soc. 2005, 127, 8775-8784.

[15] W. Al-Soufi, B. Reija, S. Felekyan, C. A. Seidel, M. Novo, ChemPhysChem 2008, 9, 1819-1827.

[16] O. G. Berg, P. H. von Hippel, Annu. Rev. Biophys. Biophys. Chem. 1985, 14, 131-158.

[17] D. Vuzman, A. Azia, Y. Levy, J. Mol. Biol. 2010, 396, 674-684. 
[18] J. Bordello, M. Novo, W. Al-Soufi, J. Colloid Interface Sci. 2010, 345, 369-376.

[19] M. Novo, D. Granadero, J. Bordello, W. Al-Soufi, J. Inclusion Phenom. Macrocyclic Chem. 2011, 1-10.

[20] (a) W. D. Wilson, F. A. Tanious, H.J. Barton, R.L. Jones, K. Fox, R.L. Wydra, L- Strekowski, Biochemistry 1990, 29, 8452-8461. (b) F. A Tanious, J. M. Veal, H. Buczak, L. S. Ratmeyer, W. D. Wilson, Biochemistry 1992, 31, 3103-3112. (c) T. M. Lohman, CRC Crit. Rev. Biochem. 1986, 191-245.

[21] S. Neidle, Nat. Prod. Rep. 2001, 18, 291-309.

[22] D. Granadero, J. Bordello, M. J. Pérez-Alvite, M. Novo, W. Al-Soufi, Int. J. Mol. Sci. 2010, 11, 173-188. 
Entry for the Table of Contents (Please choose one layout)

\section{DNA Binding Dynamics}

Jorge Bordello, Mateo I. Sánchez, Prof. Dr. M. Eugenio Vázquez, Prof. Dr. José L. Mascareñas, Prof. Dr. Wajih Al-Soufi and Prof. Dr. Mercedes Novo*

Page - Page

Single-Molecule Approach to DNA Minor-Groove Association Dynamics

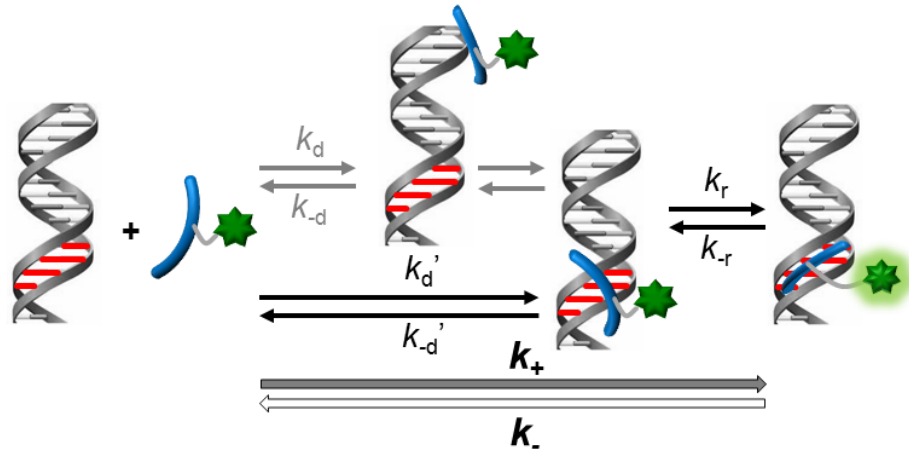

Tight Fit: Fluorescence Correlation Spectroscopy reveals that the dynamics of the association process of the bis-benzamidine minor-groove binder BBA-OG to dsDNA is not controlled by diffusion, but by the insertion of the binder into the groove at the specific site. The association process has an important effect on the specificity to AT sites. 